\title{
COMUNICAÇÃO
}

\section{PODA DE RECUPERAÇÃO EM TANGERINEIRA 'PONKAN' NO MUNICÍPIO DE PERDÕES-MG}

\author{
Prunning recuperation of tangerine 'Ponkan' in the county of Perdões-MG
}

\author{
Vander Mendonça², José Darlan Ramos ${ }^{3}$, Sebastião Elviro de Araújo Neto², \\ José Carlos Moraes Rufini ${ }^{2}$, Tiago Chaltein Almeida Gontijo ${ }^{4}$, Edney Paulo Carrijo ${ }^{4}$
}

\section{RESUMO}

Objetivou-se com esta pesquisa testar o efeito da poda de topo no rebaixamento da copa e poda da parte baixa da planta (saia) na recuperação da tangerineira 'Ponkan' com 12 anos de idade, altura de 5 metros, espaçadas de 6 x 4 m e enxertadas sobre limoeiro 'Cravo'. O experimento foi conduzido na Fazenda Vito Crincoli, localizada no município de Perdões, MG. O delineamento experimental utilizado foi em blocos ao acaso, em esquema fatorial 4 x 2, sendo poda do topo (sem poda, poda a 3,$0 ; 2,5$ e $2,0 \mathrm{~m}$ ) e poda da saia (sem e com a poda) com quatro repetições. A parcela foi constituída de três plantas. A produção da tangerineira, após o primeiro ano da poda, reduziu-se em função sua intensidade. Os diferentes tipos de poda não alteraram a qualidade dos frutos.

Termos para indexação: Citrus reticulata Blanco, qualidade, produção.

\begin{abstract}
The aim of this work was to verify the effect of top pruning for the top reduction and the skirt pruning for the recuperation of tangerine Ponkan trees 12 years old, 5 meters of height, plants with $6 \times 4$ meters of distance and grafted over 'Cravo' lemon tree. The experiment was conducted at Vito Crincoli Farm, located at county of Perdões-MG Brazil. The experimental design was in randomized blocks, in two factors arrangement (4x2) composed by: top pruning (without pruning and pruning at levels 3,0; 2,5; 2,0 meters of height) and skirt pruning (with and without pruning), with fours replicates. Each plot was composed by three plants. The production of the tangerine trees after the first year of pruning decreased because of the pruning intensity. The different types of pruning did not change the fruits quality.
\end{abstract}

Index terms: Citrus reticulata Blanco, quality, production.

(Recebido para publicação em 26 de maio de 2003 e aprovado em 3 de setembro de 2003)

A tangerineira 'Ponkan', entre os cítricos, é a principal cultivar utilizada nessa região, correspondendo a $86,9 \%$ da área plantada, e o município de Perdões destaca-se pela produção diferenciada e qualidade de seus frutos, Rossi Júnior (1999).

A utilização de tecnologias modernas em nossa região ainda é pouco utilizada pelos citricultores, assim, a maioria prefere cultivar a tangerineira de maneira tradicional, propiciando o aumento do volume das copas, sem se preocupar com o crescimento desordenado da planta, o que dificulta e encarece os tratos culturais, principalmente a colheita.
A prática da poda já vem sendo utilizada em alguns países com sucesso, favorecendo a maior luminosidade, com redução do porte das plantas melhorando a qualidade dos frutos. Os métodos mais utilizados para controle do tamanho e estrutura da copa são a poda seletiva de ramos, poda de topo (flat-topped e gable-topped) e poda lateral (Hedging) (PETTO NETO, 1991).

Em plantas cítricas, a redução na produtividade é tanto maior quanto mais severas e freqüentes forem as podas, com grande redução na produtividade imediatamente nas safras subseqüentes (STUCHI, 1994; FALLAHI e KILBY, 1997).

\footnotetext{
1. Trabalho financiando com bolsa da CAPES.

2. Engenheiros Agrônomos, M.Sc., Doutorandos-Agronomia/Fitotecnia - Universidade Federal de Lavras/ UFLA, Caixa Postal 37 - $37200-000$ - Lavras, MG, vander@ufla.br, bolsistas da CAPES

3. Engenheiro Agrônomo, Dr., Professor do Departamento de Fitotecnia/UFLA, darlan@ufla.br, bolsista do CNPq

4. Estudantes de Graduação em Agronomia/UFLA, tiagocgontijo@bol.com.br, bolsista do CNPq
} 
Em poda de topo, quanto mais se rebaixa a altura, menores são as produções acumuladas, e quando feita anualmente, promove uma baixa produção acumulada, quando comparada às freqüências bienal, trienal e sem poda (STUCHI, 1994). Em tangor Murcote, a remoção de $50 \%$ de sua copa causou redução significativa da produtividade nos três primeiros anos, mas se recuperou após o $4^{\circ}$ ano (CASTLE, 1983).

Fallahi e Kilby (1997) também alertam para o efeito das podas drásticas, que podem comprometer severamente a produtividade nas safras subsequientes. Porém, Petto Neto (1991) observa que as plantas podadas podem comprometer a produção ou não produzir fruto algum na safra seguinte, mas se bem tratadas, a partir do segundo ano da poda, a produção poderá ser boa e com frutos de melhor qualidade.

Conduziu-se esta pesquisa com o objetivo de testar o efeito da poda de topo no rebaixamento da copa e poda da parte baixa da planta (saia) em tangerineira 'Ponkan', visando à recuperação da planta.

O experimento foi conduzido na Fazenda Vito Crincoli, localizada no município de Perdões, MG, em plantas de tangerineira (Citrus reticulata Blanco cv. Ponkan) com 12 anos de idade, altura média de 5 metros, espaçadas de $6 \times 4 \mathrm{~m}$ e enxertadas sobre limoeiro 'Cravo'.

O delineamento experimental utilizado foi em blocos ao acaso, em esquema fatorial $4 \mathrm{x}$ 2, sendo o fator principal poda do topo (sem poda, poda a 3,$0 ; 2,5$ e 2,0 $\mathrm{m}$ de altura, medidos a partir do solo) e o fator secundário poda da parte baixa da planta (saia) (sem e com a poda). Os dois tipos de podas foram feitas na data 12/11/01. A parcela experimental foi constituída de cinco plantas, das quais apenas as três plantas centrais constituíram a parcela útil. Cada tratamento foi repetido quatro vezes.

Foram avaliados: diferença na altura da planta e diferença em relação ao diâmetro da copa. Para essas características, foram feitas duas medições: uma da altura (medida a partir do solo) e outra do diâmetro da copa das plantas, medido a partir da formação da copa até o topo da planta, seis meses após a poda, comparando com as medições realizadas no primeiro dia da poda. Na colheita da safra seguinte (04/06/02), cerca de sete meses após a poda, os frutos de cada tratamento foram separados, contados e pesados para avaliação do peso médio do fruto $(\mathrm{g})$, número de fruto por planta e estimativa de produtividade (t/ha). A qualidade do fruto foi avaliada em uma amostragem de cinco frutos por planta, mediante características de sólidos solúveis totais - SST (\%), acidez total titulável -ATT (\%), ratio (SST/AAT) e diâmetro do fruto $(\mathrm{cm})$.

O efeito da interação entre a poda do topo e da saia foi significativo para as características, número de frutos/planta, produção/planta, produtividade e redução no diâmetro da copa. Para o crescimento da planta, apenas o efeito da poda do topo foi significativo e as demais características avaliadas não foram influenciadas pelas diferentes intensidades da poda (Tabela 1).

A produção por planta e produtividade foi reduzida em função da intensidade da poda do topo, porém, a maior produção e produtividade foram alcançadas sem a poda de topo, associada com a poda da saia, com uma produtividade de 39,9 t/ha, mostrando o efeito benéfico quando se utiliza somente esse tipo de poda na planta, fato observado em todos os tratamentos, com exceção apenas da poda de topo a $2 \mathrm{~m}$ associada a esse mesmo tipo, que reduziu a produção para apenas 4,4 t/ha. A redução na produção apresentada nos tratamentos com podas drásticas em parte pode ser explicada pela época das podas, já que foram realizadas no mês de novembro, quando a planta já apresentava frutos em desenvolvimento, eliminando, assim, grande parte da produção. Em poda de topo, quanto mais se rebaixa a altura, menores são as produções acumuladas e, quando feita anualmente, promove uma baixa produção acumulada quando comparada às freqüências bienal, trienal e sem poda (STUCH, 1994). Porém, o rebaixamento severo da copa propicia o aumento do tamanho do fruto (FALLAHI e KILBY, 1997). Petto Neto (1991) afirma que em plantas bem tratadas, após dois anos, a produção se estabiliza, com melhorias na qualidade dos frutos. Outro resultado que se destacou na produção foi da poda de topo a 3,0 $\mathrm{m}$ associada à poda da saia, obtendo-se $64,57 \mathrm{~kg}$ de frutos por planta. 
TABELA 1 - Produção, número de frutos por planta, peso médio do fruto, produtividade, peso médio do fruto, diâmetro do fruto, SST, Ratio, ATT e diferença em diâmetro da copa (DDC) de plantas de tangerineira 'Ponkan' após poda do topo e poda da parte baixa da planta (saia). Lavras-MG, 2003.

\begin{tabular}{ccc}
\hline & \multicolumn{2}{c}{ Produção* (kg/planta) } \\
\hline Poda Topo & \multicolumn{2}{c}{ Poda Saia } \\
& Sem poda & Com poda \\
Sem poda & 63,90 a B & 96,13 a A \\
Poda a 3,0 m & 43,00 ab A & 64,57 b A \\
Poda a 2,5 m & 28,00 b A & 28,23 c A \\
Poda a 2,0 m & 23,73 b A & 10,53 c A \\
\hline C.V $(\%)$ & \multicolumn{2}{c}{33,41} \\
\hline
\end{tabular}

\begin{tabular}{ccc}
\hline Poda Topo & \multicolumn{2}{c}{ Poda Saia } \\
& Sem poda & Com poda \\
Sem poda & 302,8 a A & 330,5 a A \\
Poda a 3,0 m & 176,8 b B & 270,3 a A \\
Poda a 2,5 m & 132,5 b A & 141,3 b A \\
Poda a 2,0 m & 118,0 b A & 44,0 b A \\
\hline C.V $(\%)$ & & 28,78 \\
\hline
\end{tabular}

\begin{tabular}{ccc}
\hline & \multicolumn{2}{c}{ Produtividade (t/ha) } \\
\hline Poda Topo & \multicolumn{2}{c}{ Poda Saia } \\
& Sem poda & Com poda \\
Sem poda & 26,6 a B & 39,9 a A \\
Poda a 3,0 m & 17,9 a A & $25,6 \mathrm{~b} \mathrm{~A}$ \\
Poda a 2,5 m & $11,6 \mathrm{~b} \mathrm{~A}$ & $11,7 \mathrm{c} \mathrm{A}$ \\
Poda a 2,0 m & $9,9 \mathrm{~b} \mathrm{~A}$ & $4,4 \mathrm{c} \mathrm{A}$ \\
\hline C.V $(\%)$ & & 33,41 \\
\hline
\end{tabular}

Peso médio do fruto $(\mathrm{g})$

\begin{tabular}{ccc}
\hline Poda Topo & \multicolumn{2}{c}{ Poda Saia } \\
& Sem poda & Com poda \\
Sem poda & 213,25 a A & 250,25 a A \\
Poda a $3,0 \mathrm{~m}$ & 250,75 a A & 226,75 a A \\
Poda a $2,5 \mathrm{~m}$ & 208,75 a A & 200,00 a A \\
Poda a $2,0 \mathrm{~m}$ & 217,00 a A & 234,75 a A \\
\hline C.V $(\%)$ & & 18,92 \\
\hline
\end{tabular}

Continua...
Tabela 1 - Continuação...

\begin{tabular}{|c|c|c|}
\hline & \multicolumn{2}{|c|}{ Diâmetro do fruto (cm) } \\
\hline \multirow[t]{2}{*}{ Poda Topo } & \multicolumn{2}{|c|}{ Poda Saia } \\
\hline & Sem poda & Com poda \\
\hline Sem poda & 8,86 a $\mathrm{A}$ & 9,10 a $\mathrm{A}$ \\
\hline Poda a 3,0 m & 8,95 a $\mathrm{A}$ & 9,07 a $\mathrm{A}$ \\
\hline Poda a $2,5 \mathrm{~m}$ & 9,01 a $\mathrm{A}$ & 8,95 a $\mathrm{A}$ \\
\hline Poda a 2,0 m & 8,90 a A & 9,02 a $\mathrm{A}$ \\
\hline \multirow[t]{2}{*}{ C.V $(\%)$} & & 5,36 \\
\hline & \multicolumn{2}{|c|}{ SST (\%) } \\
\hline \multirow[t]{2}{*}{ Poda Topo } & \multicolumn{2}{|c|}{ Poda Saia } \\
\hline & Sem poda & Com poda \\
\hline Sem poda & 9,65 a $\mathrm{A}$ & 9,25 a $\mathrm{A}$ \\
\hline Poda a 3,0 m & 9,48 a $\mathrm{A}$ & 9,55 a $\mathrm{A}$ \\
\hline Poda a 2,5 m & 9,58 a $\mathrm{A}$ & 9,83 a $\mathrm{A}$ \\
\hline Poda a $2,0 \mathrm{~m}$ & 9,63 a $\mathrm{A}$ & 9,58 a $\mathrm{A}$ \\
\hline \multirow[t]{2}{*}{ C.V $(\%)$} & & 4,80 \\
\hline & \multicolumn{2}{|c|}{ Ratio } \\
\hline \multirow[t]{2}{*}{ Poda Topo } & \multicolumn{2}{|c|}{ Poda Saia } \\
\hline & Sem poda & Com poda \\
\hline Sem poda & 20,3 a A & 18,7 a A \\
\hline Poda a $3,0 \mathrm{~m}$ & 19,2 a $\mathrm{A}$ & 18,7 a $\mathrm{A}$ \\
\hline Poda a 2,5 m & 18,5 a $\mathrm{A}$ & 18,1 a $\mathrm{A}$ \\
\hline Poda a 2,0 m & 19,7 a $\mathrm{A}$ & 18,4 a $\mathrm{A}$ \\
\hline \multirow[t]{2}{*}{ C.V (\%) } & & 8,60 \\
\hline & \multicolumn{2}{|c|}{ ATT (\%) } \\
\hline \multirow[t]{2}{*}{ Poda Topo } & \multicolumn{2}{|c|}{ Poda Saia } \\
\hline & Sem poda & Com poda \\
\hline Sem poda & 0,480 a $\mathrm{A}$ & 0,490 a A \\
\hline Poda a 3,0 m & 0,500 a A & 0,510 a $\mathrm{A}$ \\
\hline Poda a $2,5 \mathrm{~m}$ & 0,520 a A & 0,550 a $\mathrm{A}$ \\
\hline Poda a $2,0 \mathrm{~m}$ & 0,490 a A & $0,520 \mathrm{a} \mathrm{A}$ \\
\hline \multirow[t]{2}{*}{ C.V (\%) } & & $\mathbf{8 , 3 3}$ \\
\hline & Diferença em I & ro da copa $(\mathrm{m}$ \\
\hline \multirow[t]{2}{*}{ Poda Topo } & \multicolumn{2}{|c|}{ Poda Saia } \\
\hline & Sem poda & Com poda \\
\hline Sem poda & 0,119 a A & 0,146 a $\mathrm{A}$ \\
\hline Poda a 3,0 m & 0,238 a A & 0,217 a $A$ \\
\hline Poda a 2,5 m & 0,317 a A & 0,173 a $\mathrm{A}$ \\
\hline Poda a 2,0 m & 0,223 a A & 0,412 a $\mathrm{A}$ \\
\hline C.V $(\%)$ & & 47,85 \\
\hline
\end{tabular}

*Médias seguidas de mesma letras minúscula na coluna e maiúscula na linha não diferem entre se pelo teste de Tukey à $5 \%$ de probabilidade. 


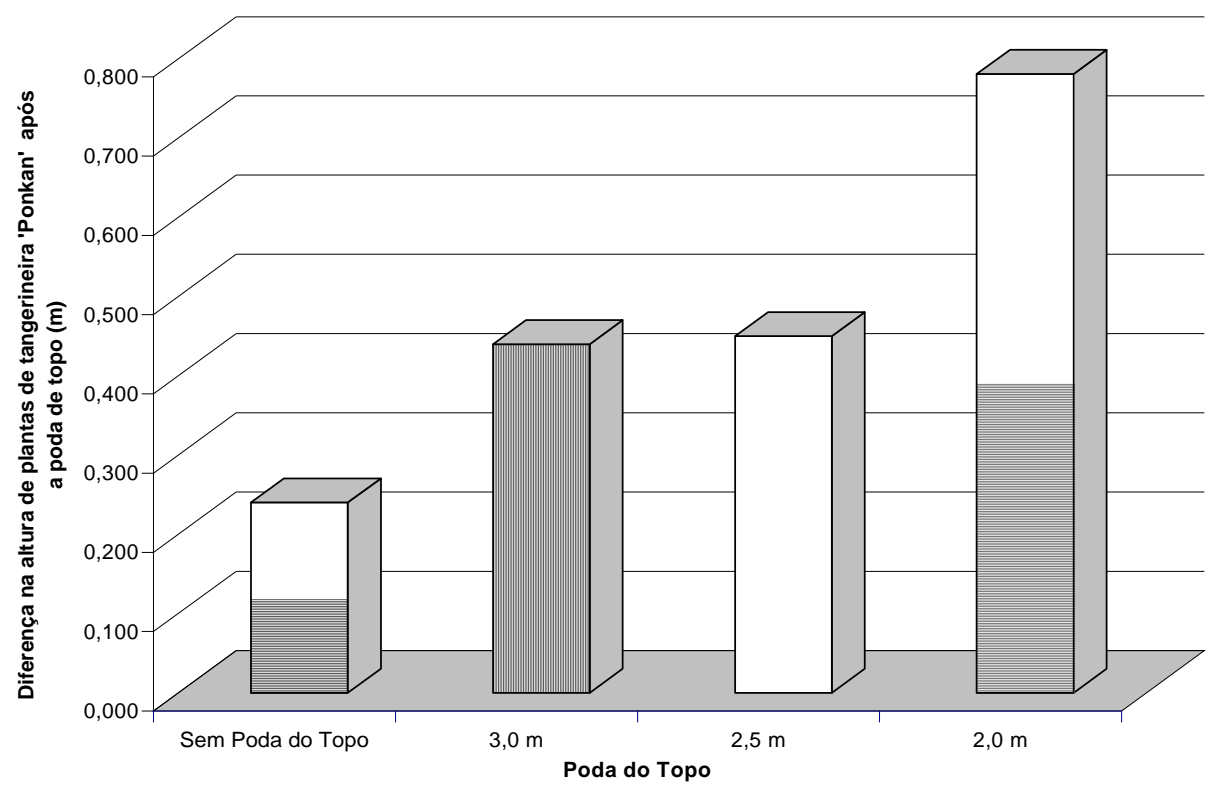

FIGURA 1 - Diferença na altura de plantas de Tangerineira 'Ponkan' após poda de topo. Lavras-MG, 2003.

Era de se esperar que o peso e o diâmetro dos frutos fossem apresentar variações em função das podas e que o tratamento com podas mais drásticas apresentassem frutos com maior peso e diâmetro, conforme relatam Fallahi e Kilby (1997), porém, estatisticamente, não foram observadas variações nessas características.

Com relação à qualidade interna dos frutos, os dois tipos de podas não provocaram alterações, com os SST, o Ratio e a ATT apresentando valores, em média, $9,55 \% 18,93$ e $0,508 \%$ respectivamente, mantendo, assim, a qualidade interna dos frutos, o que foi também observado por Morales e Davis (2000) em Tangelo 'Orlando'.

Para a característica diferença no diâmetro da copa, a poda a 2,0 m, associada à poda da saia, foi a combinação que mais influenciou, apresentando uma diferença de $412 \mathrm{~cm}$ no diâmetro em relação ao diâmetro no dia da poda. Já para a diferença no crescimento da planta, seis meses após a poda, apenas a poda do topo (Figura 1) apresentou diferença significativa, sendo a poda a $2,0 \mathrm{~m}$ a que proporcionou maior crescimento da planta em torno de $0,8 \mathrm{~m}$ após seis meses da poda.

\section{REFERÊNCIAS BIBLIOGRÁFICAS}

CASTLE, W. E. Antitranspirante and root and canopy pruning effect on mechanically transplanted eight-yearold 'Murcott' citrus trees. Journal of American Society Horticultural Science, Alexandria, v. 108, n. 6, p. 981-985, 1983.

FALLAHI, E.; KILBY, M. Tootstock and pruning influence on yield and fruit quality of 'Lisbon' lemon. Fruit Varieties Journal, [S.1.], v. 51, n. 4, p. 242-246, 1997.

MORALES, P.; DAVIS, F. S. Pruning and Skirting affect canopy microclimate, yields, and fruit quality of 'Orlando' tangelo. HortScience, Alexandria, v. 35, n. 1, p. 30-35, 2000.

PETTO NETO, A. Práticas culturais. In: VIÉGAS, R. F.; POMPEU JÚNIOR, J.; AMARO, A. S. (Eds.). Citricultura brasileira. 2. ed. Campinas: Fundação Cargill, 1991. v. 1, p. 476-492.

ROSSI, JÚNIOR, C. Aspecto da cultura de tangerinas no Sul de Minas Gerais. Laranja, Cordeirópolis, v. 20, n. 2, p. 409-417, 1999.

STUCHI, E. S. Controle do tamanho de plantas cítricas. Laranja, Cordeirópolis, v. 15, n. 2, p. 295-342, 1994. 